\title{
EL ENTORNO VIRTUAL CHAMILO EN EL DESARROLLO DE LAS COMPETENCIAS DEL MÓDULO DE OFIMÁTICA EN ESTUDIANTES DEL INSTITUTO “NORBERT WIENER” DE LINCE \\ The Chamilo virtual environment in the development of competencies in the office automation unit in students from the institute "Norbert Wiener" in Lince
}

\author{
Daniel Federico Agama Moreno ${ }^{1}$ \\ Universidad Privada Norbert Wiener, Perú
}

\section{RESUMEN}

El tipo de estudio es aplicado, de diseño cuasi-experimental, modelo pre test y post test y grupo de control, La muestra fue no probabilística, de grupos intactos $n=40$, siendo 20 estudiantes para el grupo experimental y los otros 20 del grupo control. Se tuvo como objetivo general determinar la influencia del entorno Virtual Chamilo para el logro de competencias del módulo de Ofimática en los estudiantes del IES Norbert Wiener. Para evaluar la efectividad de la plataforma Chamilo se aplicó un cuestionario de 21 preguntas para la variable dependiente desarrollo de las competencias del módulo de Ofimática, contrastándose las hipótesis planteadas, en las mejoras significativas que tuvo el grupo experimental en el desarrollo de capacidades técnicas, comunicacionales, personales y sociales, mientras que los estudiantes del grupo de control se mantuvieron en el nivel inicial. Para la prueba de hipótesis se utilizó el estadígrafo U de Mann Whitney mostrando una diferencia significativa $(\mathrm{p}=.000)$ entre ambos grupos.

Palabras Clave: Ambiente virtual de aprendizaje, entorno virtual Chamilo, competencias, tecnologias

\section{Abstract}

This is an applied study, with a quasi-experimental design, pre-test and post-test model and control group. It was a non-probability sample, with intact groups $n=40$, from which 20 students were for the experimental group and 20 for the control group. Its general objective was to determine the influence of the Chamilo Virtual Environment for the accomplishment of competencies of the Office Automation Unit in the students of the IES Norbert Wiener. To evaluate the effectiveness of the Chamilo platform a questionnaire of 21 questions for the dependent variable development of the competencies in the Office Automation Unit was applied, by contrasting the stated hypotheses, in the significant improvements that the experimental group had in the development of technical, communicative, personal and social capabilities, while the students in the control group remained at the initial level. In order to test the hypothesis, the U Mann-Whitney test was used, showing a significant difference $(\mathrm{p}=.000)$ between both groups.

Keywords: Virtual learning environment, Chamilo virtual environment, competencies, technologies

1 Licenciado en Computación e Informática, experto en Plataformas Virtuales, Magister en Educación, Docente de la Universidad Privada Norbert Wiener. daniel_agama@gmail.com 
El entorno virtual Chamilo en el desarrollo de las competencias del módulo de ofimática en estudiantes del instituto "Norbert Wiener" de Lince

\section{INTRODUCCIÓN}

En la actualidad los diversos profesionales que imparten enseñanza a nivel superior, se han visto obligados a utilizar herramientas tecnológicas como apoyo a su rol docente, haciendo que estas formen parte inseparable de su labor pedagógica, más aun ahora que se cuenta con plataformas virtuales de software libre y de acceso gratuito, $y$ esto debido a que muchos de los estudiantes han dejado de utilizar el lápiz y papel para sus anotaciones, pasando a utilizar los procesadores de textos, block note, aplicaciones informáticas, utilizar las grabaciones como medios para su aprendizaje y complementar con los mismos fuera de sus horarios de clases, debido a estos cambios radicales que se están presentando es que se torna necesario que los docentes puedan utilizar herramientas que les permitan brindar a sus estudiantes contenidos específicos de la asignatura que imparten de una manera sincrónica como asincrónica, disponible las 24 horas del día, en el lugar y espacio accesible para ellos, rompiendo las barrera de tiempo y espacio, que en muchos casos ha sido un limitante, para que ellos sigan desarrollando competencias idóneas a su formación profesional, sumado a ello el cambio de roles que ha sufrido el docente, como lo menciona Dellepiane (2013), al pasar este a ser un creador de hábitos de búsqueda, selección de información, desarrollar experiencias colaborativas, intercambio, retroalimentación y encaminar el proceso de enseñanza aprendizaje al saber hacer apropiándose de las herramientas tecnológicas, Valenzuela-Zambrano y Pérez-Villalobos (2013) al respecto refieren que el docente es quien está orientado a diseñar actividades, a través de estrategias de enseñanza, en la cual el estudiante identifique sus errores en procesos y los solucione, así como potenciar sus habilidades y destrezas, intentando monitorizar, regular y controlar su cognición, comportamiento y motivación en el logro de su aprendizaje, para lo cual debe utilizar los medios digitales que tiene a su disposición. Ardila, Ruíz y Castro (2015) por su parte refieren que en la relación enseñanza aprendizaje se debe incorporar el uso de herramientas de comunicación sincrónicas y asincrónicas, como son las plataformas virtuales de código abierto, por el potencial que tienen y se adaptan a las necesidades de las universidades. Lo indicado anteriormente no dista mucho en nuestra realidad, ya que el impartir docencia en este nuevo escenario donde las tecnologías están inmersas en la vida cotidiana y el quehacer del día a día de nuestros estudiantes, es que se torna indispensable utilizar esta herramienta tecnológica en el proceso de enseñanza aprendizaje, para lo cual se consideró como objetivo general determinar la influencia del entorno virtual Chamilo en el logro de competencias del módulo de Ofimática en los estudiantes del IESP Norbert Wiener del distrito de Lince, como objetivos específicos: (i) Determinar la influencia que produce la utilización del entorno Virtual Chamilo en el logro de capacidades técnicas del módulo de Ofimática (ii) determinar la influencia que produce la utilización del entorno Virtual Chamilo en el logro de capacidades comunicacionales del módulo de Ofimática (ii) determinar la influencia que produce la utilización del entorno Virtual Chamilo en el logro de capacidades personales y sociales del módulo de Ofimática.

\section{Antecedentes}

El uso de plataformas virtuales en educación para la enseñanza ha venido emergiendo paulatinamente en todos los niveles de educación, en el caso de las universidades esto ha conllevado a realizar cambios en los procedimientos pedagógicos, como se puede observar en los estudios realizados por Fernández (2009), quien afirma que la mayoría de los docentes del espacio Europeo de educación superior utiliza los entornos virtuales de aprendizaje para la formación en cursos o asignaturas universitarias de primer y/o segundo ciclo, el $48,2 \%$ considera que estas herramientas permiten organizar y gestionar una asignatura, el $65,8 \%$ indica que contribuyen a facilitar la autoformación, formación colaborativa y cooperativa, el 52,1\% están de acuerdo que estos entornos ayudan al estudiante a continuar en el proceso de enseñanza aprendizaje en los nuevos modelos europeos y el 51,9\% afirman que estos medios favorecen la adaptación al espacio Europeo de educación superior, por su parte Maldonado (2012) indica que los estudiantes están de acuerdo en realizar cursos 
cortos por medio de la teleformación a través de la aplicación de entornos virtuales de aprendizaje; Forestello y Arónica (2013) mencionan que el uso de los entornos virtuales por parte de los docentes dependerá de las creencias y supuestos que estos tengan de cómo las tecnologías son un medio para el apoyo de ampliar los conocimientos a los estudiantes. Por su parte Benitez, Cruces y Sarrión (2011) mencionan que desde la experiencia de la incorporación de la plataforma virtual para la enseñanza en la modalidad presencial ha facilitado la labor docente y la participación activa de los estudiantes implicándolos en la tarea de valoración de las actividades realizadas por ellos mismos y de otros compañeros.

\section{ENTORNO VIRTUAL DE}

\section{APRENDIZAJE}

El docente que imparte catedra a nivel universitario ha tenido que buscar medios alternativos como estrategias en el proceso de enseñanza aprendizaje, los cuales le permitan acercarse más aun a sus estudiantes en la no presencialidad, esto ha conllevado a la implementación de entornos virtuales de aprendizaje, Peña y Avendaño (2006) mencionan que estos entornos están inmersos en aplicaciones informáticas diseñadas para la comunicación pedagógica tanto para la modalidad presencial o a distancia o en ambas. Para los autores Ibáñez, Delgado y Aylett, (2006, p. 47) es un espacio de representaciones tridimensionales, computarizadas, la cual permite a los usuarios interactuar libremente en tiempo real. Ospina (2009) los refiere como espacios educativos, que están alojados en una web e integrados por herramientas informáticas, que hacen viable la interacción didáctica y que deben poseer cuatro características básicas, la primera es ser un ambiente electrónico, la segunda pertenecer a una red con acceso remoto a los contenidos, la tercera es que los programas informáticos que la conforman sirven de soporte para las actividades formativas de docentes y estudiantes y la cuarta es que la relación didáctica es mediada por las tecnologías digitales, sin necesidad de que docentes y estudiantes coincidan en el espacio o en el tiempo. Por su parte Salinas (2011, p.25) enfatiza que estos pueden ser llamados ambientes virtuales, entornos, mundos virtuales. Ferreira y Sanz (2012 p. 94) refieren que los entornos virtuales de enseńanza y aprendizaje son un conjunto de aplicaciones informáticas diseñadas para la utilización de internet con fines educativos. En el caso de Valenzuela-Zambrano y Pérez-Villalobos (2013) lo refieren como plataformas de enseñanza virtuales, que son programas de aplicación de software instalados en un servidor, utilizado para administrar, distribuir y controlar las actividades de formación, pudiendo ser estas comerciales o de acceso libre.

Para Ardila, Ruíz y Castro (2015, p. 58) un entorno virtual de aprendizaje es un sistema de gestión de aprendizaje alojado en un servidor desde el cual se proporcionan servicios y herramientas para el apoyo en formación de los estudiantes a través de evaluaciones, actividades y que se complementan con medios de comunicación como foros, chats, juegos y videoconferencias y tienen funciones establecidas como gestionar y administrar usuarios, cursos y herramientas de comunicación.

\section{Características del entorno virtual de aprendizaje}

La evolución que ha tenido los entornos virtuales de aprendizaje, han conllevado a describir ciertas características que estos deben poseer para ser implementados en el portal de la universidad para el proceso de enseñanza aprendizaje, como lo señala Ardila, Ruíz y Castro (2015) al evaluar la plataforma idónea para la universidad de Boyacá, resaltan que esta debe considerar la Inclusión, para personas con necesidades especiales, población vulnerable o aquellas que residen en regiones con deficiente conectividad; la evaluación formativa, facilidad de la plataforma para retroalimentar a tutores y educandos; evaluación continua, facilidad de revisión de cursos; aprendizaje efectivo, flexibilidad para personalizar el ambiente virtual por parte del usuario, así como el acompañamiento docente; accesibilidad, facilidad en la revisión de contenidos y recursos; usabilidad, simplicidad que tiene la plataforma para ser usada; herramientas, el tener herramientas para ser usada por los usuarios de manera sincrónica, asincrónica y permitir la evaluación; durabilidad, posibilidad de realizar cambios sin afectar a los usuarios; 
El entorno virtual Chamilo en el desarrollo de las competencias del módulo de ofimática en estudiantes del instituto "Norbert Wiener" de Lince

empaquetamiento, formato en que se distribuye la aplicación; confiabilidad, el poder ver la frecuencia de errores de la plataforma; funcionalidad, capacidad para la gestión y administración de los cursos; eficiencia, inmediatez para que los usuarios perciban cambios en la plataforma; reutilización, posibilidad de volver a ser utilizado de manera parcial o total la estructura de un curso; y la interoperabilidad y portabilidad, el poder intercambiar información con otro y capacidad de funcionar en diferentes sistemas operativos y navegables.

Para Valenzuela-Zambrano y Pérez-Villalobos (2013) la plataforma virtual debe poseer dos criterios, uno referido a las herramientas del docente, la cual debe permitir el generar recursos educativos, como editar cursos, exámenes, importar recursos y enrutador de recursos educativos; seguimiento y evaluación, para lo cual debe tener herramientas de seguimiento de trabajo del estudiante, herramientas de evaluación y de seguimiento de exámenes; y comunicación, asincrónicas, como el correo

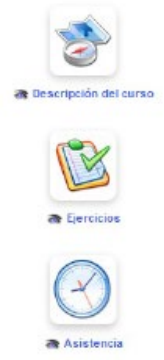
electrónico y sincrónicas como el chat, videoconferencias. La otra referida a herramientas de los estudiantes, la que debe considera la formación a través de la visualización de recursos; seguimiento y evaluación, como herramientas de auto seguimiento, autoevaluación, de realización de exámenes y de revisión de exámenes; comunicación entre usuarios, asincrónica, como el correo electrónico y sincrónico como el chat y videoconferencias.

\begin{tabular}{|c|c|}
\hline Iconos & Funciones \\
\hline 0 & Actualizar la configuración de la actividad (título, ponderación, etc.) \\
\hline$\approx$ & $\begin{array}{l}\text { Quitar una actividad de la evaluación. Las actividades presenciales serán } \\
\text { eliminadas, y las actividades en línea serán simplemente quitadas de la } \\
\text { evaluación }\end{array}$ \\
\hline क & $\begin{array}{l}\text { Cambiar la visibilidad de los resultados de la actividad en el "libro de } \\
\text { evaluación" del estudiante. }\end{array}$ \\
\hline 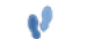 & Mostrar el historial de cambios en la configuración de la actividad \\
\hline
\end{tabular}

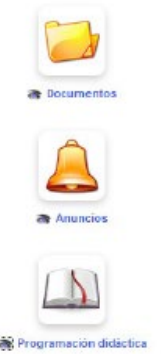

permitir que se organice y planifique los cursos creados, al poder importar o crear documentos, contenidos, construir exámenes con calificación automática y retroalimentación si es necesaria, configurar la entrega de trabajos virtuales, comunicar a través del foro o chat, publicar anuncios, agregar enlaces, crear grupos de trabajos, realizar videoconferencias, utilizar glosarios, agenda, gestionar un proyecto a través de la herramienta blog, hacer seguimientos a los estudiantes en los cursos, registrar asistencia y elaborar un diario de clases para verificación del progreso de los estudiantes. En las siguientes figuras se presentan algunas herramientas que son utilizadas para la creación de un curso.

Figura 1. Herramientas de creación de contenidos (Chamilo, s.f)

\section{El entorno virtual Chamilo}

Chamilo (s.f) refiere que Chamilo Learning Management System (LMS) es un sistema de gestión del aprendizaje, con software de acceso libre, diseñado para proporcionar ayuda y apoyo dentro de la propia aplicación, el cual tiene una serie de herramientas que le permiten al docente crear un entorno de aprendizaje eficiente y personalizado, ajustado a las características y objetivos del curso o las competencias que se desea desarrollar en los estudiantes, por la flexibilidad que posee, al
Figura 2. Herramientas para gestionar actividades (Chamilo, s.f.)

\section{Competencias del módulo de ofimática}

Arnold y Schüssler (2001, p. 117) afirman que el término competencia se refiere a la capacidad de una persona para actuar en determinada situación y la habilidad que esta tiene para ejecutar tareas y roles que son requeridos en función de estándares 
esperados. UPV (2006), Pazo y Tejada (2012) hacen referencia a dos tipos de competencias, una de ellas es la competencia genérica, que tiene relación con aspectos generales, comunes y exigidas en todo el proceso de formación en los diversos cursos que se imparten, es el saber ser y saber estar y la otra es la competencia específica, que son saberes propios de una profesión es el saber hacer en campos específicos de la especialidad, adicional a lo mencionado Escalante, patrón y Argüelles (2011, p.93) enfatizan que las competencias son capacidades que la persona desarrolla en forma gradual y a lo largo de todo el proceso educativo y son evaluadas en diferentes etapas. Para Vargas-D'Uniam, et al (2014) hace referencia a la competencia digital considerándola como aquella que corresponde a la capacidad de entender, evaluar y utilizar en diversos contextos los medios digitales y las herramientas de comunicación con la utilización de las TIC; desde esta perspectiva Amador, et al (2015) enmarca a la competencia en técnicas y tecnológicas, siendo estas la apropiación de las TIC, el manejo y desarrollo de destrezas para navegar, apropiarse de las WEB 2.0 y 3.0. Por su parte Figueras, et al (2016) hace referencia a las competencias básicas, las cuales son adquiridas en la formación de los estudiantes para su realización personal, el poder desenvolverse en el mundo actual y ser aptos para desempeñar una profesión, para lo cual debe desarrollar ciertas habilidades y destrezas, como la comunicación lingüística, competencia matemática, en ciencia y tecnología, competencia digital, aprender a aprender, competencias sociales y cívicas, sentido de iniciativa y espíritu emprendedor, conciencia y expresiones culturales.

De estas diversas acepciones en el presente estudio se ha considerado a la competencia del módulo de ofimática como capacidades, la cual incluye a las capacidades técnicas, capacidades comunicaciones y capacidades personales y sociales, considerando lo expuesto por Marqués (2000) en cuanto a capacidades técnicas, quien refiere que es el conocimiento básico de las características y terminología de los sistemas informáticos, la gestión de sistemas tecnológicos aplicados a la educación, la cual permite que el estudiante deba saber cómo conectar los periféricos de manera adecuada; encender y apagar los equipos correctamente; ma- nejar los sistemas operativos y sus funciones más comunes; y saber hacer un mantenimiento básico a los diferentes dispositivos, saber usar el procesador de textos, manejando sus funciones más importantes, utilizar las hojas de cálculo con sus funciones más comunes y la creación de gráficos estadísticos, el manejo de bases de datos (creación, mantenimiento, consultas, informes), elaboración de presentaciones multimedia, utilización de lenguajes de autor y entornos específicos para la creación de materiales educativos digitales; uso de tutoriales digitales y de las ayudas que proporcionan los mismos programas, la creación, la captura y edición de imágenes digitales; uso del escáner y de la cámara de video y fotográfica; conocimientos básicos del lenguaje hipermedia, elaboración de Sitios Web, creación de Páginas, Blog y Wikis, navegación en Internet, uso de buscadores y meta buscadores, búsqueda y selección critica de información; uso del correo electrónico, foros telemáticos y lo definido por Van Arcken (2010), con relación a competencia técnica al conocimiento y habilidad de una persona en realizar cualquier tipo de proceso con el uso de herramientas y programas tecnológicos.

En cuanto a las capacidades comunicacionales se ha considerado a Trelles (2009) que las define como un conjunto de procesos para la construcción de significados compartidos entre los miembros de una organización, al ser este un proceso de construcción de significados que favorece el logro de niveles de participación cualitativamente superiores y la interacción colaborativa. Con relación a las capacidades personales y sociales se ha considerado lo indicado por Mora-Valentín y Ortiz-De-Urbina-Criado (2015) quienes mencionan que las capacidades personales es la capacidad de trabajar en equipo, trabajo en equipo de carácter interdisciplinar, trabajo en un contexto internacional, habilidad en las relaciones personales, capacidad para trabajar en entornos diversos y multiculturales, capacidad crítica y autocrítica, compromiso ético en el trabajo, trabajar en entornos de presión y las capacidades sociales según lo mencionado por Rodrigues, Simóes y Ferreira (2013) es el comportamiento agradable, cooperativo y el deseo de participar en tareas compartidas con sus pares. 
El entorno virtual Chamilo en el desarrollo de las competencias del módulo de ofimática en estudiantes del instituto "Norbert Wiener" de Lince

El módulo como lo refiere Alvites-Huamaní y Bayona (2014) es una secuencia articulada de unidades y actividades de aprendizaje que en un determinado tiempo permite alcanzar los objetivos propuesto para el desarrollo de capacidades, el cual se realiza a través de una serie de sesiones de formación, el cual contiene los contenidos temáticos. En cuanto a ofimática este se refiere a la automatización de las comunicaciones y procesos que se realizan en una oficina; y permite agilizar las funciones de gestión de estas (MAD, 2005).

Las competencias del módulo de ofimática en este estudio se refieren al conjunto de desarrollo de capacidades técnicas y comunicativas en cuanto al uso de medios informáticos, básicamente con el uso del entorno virtual de aprendizaje Chamilo.

\section{Materiales y Métodos}

\section{Participantes}

La población fue un total de 64 estudiantes del II ciclo del Instituto Educación Superior Norbert Wiener del distrito de Lince pertenecientes a tres secciones del turno de la mañana (sección $1=20$, sección $2=20$ y sección $3=24$ ) e inscritos en el módulo de Ofimática, desarrollado en el primer semestre del 2013. La muestra fue no probabilística de tipo intencional con grupos intactos, lo cual a los sujetos no se le asignaron al azar, si no ya estaban formados antes del experimento, (Hernandez, et al, 2010)

Tabla 1

Muestra de los grupos control y experimental

\begin{tabular}{|c|c|c|}
\hline Sección & Grupo & $\begin{array}{l}\text { Cantidad de } \\
\text { estudiantes }\end{array}$ \\
\hline 1 & Control & 20 \\
\hline \multirow[t]{2}{*}{2} & Experimental & 20 \\
\hline & Total & 40 \\
\hline
\end{tabular}

Fuente: Dirección Académica del IES Norbert Wiener.

\section{Instrumento}

Para la recolección de datos se elaboró el instrumento "prueba de competencias del módulo de Ofimática”, el cual estuvo estructurado con 21 ítems, realizándose la validación por juicio de expertos, obteniendo un promedio de $97.3 \%$, y la confiabilidad con el coeficiente de consistencia interna alfa de Cronbach, que dio $\alpha=.967$ (alta confiabilidad), el cual se aplicó antes y después del experimento.

\section{Tabla 2}

Dimensiones de la variable Competencias del Módulo de Ofimática

\begin{tabular}{|c|c|}
\hline Dimensiones & Indicadores \\
\hline \multirow[t]{5}{*}{$\begin{array}{l}\text { Capacidades } \\
\text { Técnicas }\end{array}$} & $\begin{array}{l}\text { Desarrolla capacidades cognitivas previs- } \\
\text { tas }\end{array}$ \\
\hline & $\begin{array}{l}\text { Desarrolla destrezas motoras para operar } \\
\text { herramientas y maquinarias. }\end{array}$ \\
\hline & $\begin{array}{l}\text { Identifica materiales para realizar activida- } \\
\text { des productivas }\end{array}$ \\
\hline & $\begin{array}{l}\text { Identifica equipos para realizar actividades } \\
\text { productivas }\end{array}$ \\
\hline & $\begin{array}{l}\text { Ejecuta acciones de control en: recursos, } \\
\text { procesos y productos. }\end{array}$ \\
\hline \multirow[t]{3}{*}{$\begin{array}{l}\text { Capacidades } \\
\text { Comunicacionales }\end{array}$} & $\begin{array}{l}\text { Utiliza medios informáticos para elabora- } \\
\text { ción de informes técnicos y comerciales }\end{array}$ \\
\hline & $\begin{array}{l}\text { Adquiere y utiliza datos y/o programas } \\
\text { informáticos }\end{array}$ \\
\hline & $\begin{array}{l}\text { Organiza e interpreta información median- } \\
\text { te equipos de computación }\end{array}$ \\
\hline \multirow[t]{3}{*}{$\begin{array}{l}\text { Capacidades per- } \\
\text { sonales y sociales }\end{array}$} & $\begin{array}{l}\text { Capacidad para trabajar en equipo y asu- } \\
\text { mir el liderazgo. }\end{array}$ \\
\hline & $\begin{array}{l}\text { Actúa con criterio lógico ante situaciones } \\
\text { imprevistas tomando decisiones oportunas. }\end{array}$ \\
\hline & $\begin{array}{l}\text { Mantiene relaciones fluidas con el grupo } \\
\text { de trabajo }\end{array}$ \\
\hline
\end{tabular}

Fuente: Dirección Académica del IES Norbert Wiener.

\section{Tipo y Diseño}

El tipo de investigación es cuantitativa, pues los datos se recogieron con base en una escala de medición numérica, para analizarse mediante la estadística. El diseño es cuasi-experimental, con pre prueba, posprueba y grupo de control se realizó a través de la administración de prueba a los grupos que componen el experimento, asignándose los participantes al azar, a quienes se les aplica simultáneamente la pre prueba; un grupo recibe el tratamiento experimental y otro no (es el grupo control); por último se le administra, también simultáneamente, una pos prueba." (Hernández, et al. 2010). 


\section{Procedimiento}

Para la ejecución de la investigación se solicitó la autorización de la dirección de estudios académicos del instituto, previa a la elaboración del módulo y de las sesiones de clases, asimismo se solicitó el consentimiento informado de los participantes, para el caso del grupo experimental, ya que ellos eran quienes tendrían la aplicación de esta nueva estrategia. A ambos grupos se les proporciono los mismos contenidos temáticos, brindándose de acuerdo a la programación establecida, al grupo de control se impartió clases de manera tradicional y al grupo experimental la aplicación del entorno virtual de aprendizaje Chamilo, durante un semestre académico que dura el módulo de ofimática.

$\mathrm{Al}$ inicio del curso se realizó un pretest a ambos grupos y al concluir el curso se realizó el post test para verificar las diferencias entre ambos grupos. y experimental indicaron que, en el pretest de la variable competencias del módulo de Ofimática (tabla 3), no hubo diferencias significativas $(p>$ $.05)$ entre los grupos; en cambio, en el postest, la prueba indicó que entre los grupos de control y experimental hubo una diferencia muy significativa $\left({ }^{* *} p<.01\right)$, como consecuencia de la utilización del entorno virtual Chamilo.

\section{Tabla 3}

Resultados de la prueba de hipótesis para la diferencia entre los grupos de control y experimental en las competencias del módulo de Ofimática

Logro de competencias del módulo de Ofimática

\begin{tabular}{|c|c|c|c|c|c|}
\hline \multirow[b]{2}{*}{ Fase } & \multirow[b]{2}{*}{ Grupo } & \multirow[b]{2}{*}{ Media } & \multirow{2}{*}{$\begin{array}{l}\text { Desviación } \\
\text { estándar }\end{array}$} & \multicolumn{2}{|c|}{ U de Mann Whitney } \\
\hline & & & & $\mathbf{U}$ & $p$-valor \\
\hline \multirow{2}{*}{ Pretest } & Control & 20.70 & 2.003 & 138.000 & 0.096 \\
\hline & Experimental & 26.65 & 2.477 & & \\
\hline \multirow{2}{*}{ Postest } & Control & 28.90 & 2.532 & 0.000 & 0.000 \\
\hline & Experimental & 92.40 & 8.994 & & \\
\hline
\end{tabular}

Fuente: Prueba de competencias del módulo de Ofimática, postest

\section{Resultados}

Para el contraste de hipótesis y verificar la influencia del entorno virtual de aprendizaje Chamilo en las competencias del módulo de Ofimática se utilizó la prueba U de Mann Whitney, considerándose un nivel de significancia de $.05(5 \%$ de error). La decisión se adoptó considerando si:

$p>.05$, no se rechaza la $\mathrm{H}_{0}$.

$p<.05$, se rechaza la $\mathrm{H}_{0}$ y se concluye con $\mathrm{H}_{1}$.

\section{Hipótesis General}

$\mathrm{H}_{\mathrm{a}}$ : La utilización del entorno virtual Chamilo influye significativamente en el logro de competencias del módulo de Ofimática en los estudiantes del IES Norbert Wiener del distrito de Lince.

$\mathrm{H}_{0}$ : La utilización del entorno virtual Chamilo no influye significativamente en el logro de competencias del módulo de Ofimática en los estudiantes del IES Norbert Wiener del distrito de Lince.

Los resultados de la prueba U de Mann Whitney para la diferencia entre los grupos de control
Decisión. Considerando que en el pretest la prueba U de Mann Whitney no indicó diferencias significativas entre los grupos de control y experimental, pero en el postest dio ${ }^{* *} p<.01$, al .000 de error se concluye que la utilización del entorno virtual Chamilo influye significativamente en el logro de competencias del módulo de Ofimática en los estudiantes del IES Norbert Wiener del distrito de Lince, 2013.

Según la figura 3, en el pretest de la variable logro de las competencias del módulo de Ofimática, la mediana del grupo de control (28) fue 1 punto más que la mediana del grupo experimental (27), no habiendo diferencias significativas; en cambio, en el postest, la mediana del grupo experimental (94) superó en 65 puntos a la mediana del grupo de control (29). 
El entorno virtual Chamilo en el desarrollo de las competencias del módulo de ofimática en estudiantes del instituto "Norbert Wiener" de Lince

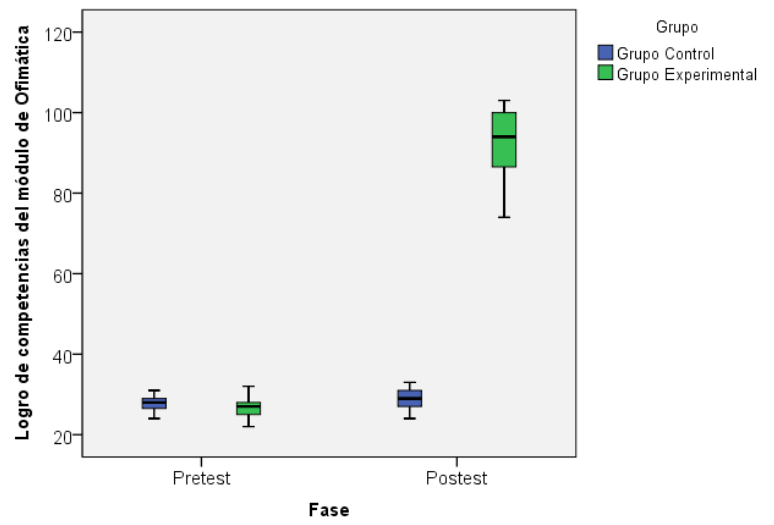

Figura 3. Diagrama de dispersión para la variable logro de competencias del módulo de Ofimática en los grupos de control y experimental, pretest y postest.

\section{Hipótesis específicas 1}

$\mathrm{H}_{1}$ : La utilización del entorno virtual Chamilo influye significativamente en el logro de capacidades técnicas del módulo de Ofimática en los estudiantes

$\mathrm{H}_{0}$ : La utilización del entorno virtual Chamilo no influye significativamente en el logro de capacidades técnicas del módulo de Ofimática en los estudiantes

Los resultados de la prueba U de Mann Whitney para la diferencia entre los grupos de control y experimental indicaron que, en el pretest de las capacidades técnicas (tabla 4), no hubo diferencias significativas $(p>.05)$ entre los grupos; por el contrario, en el postest, la prueba indicó que hubo una diferencia muy significativa $\left({ }^{* *} p<.01\right)$, como consecuencia de la utilización del entorno virtual de aprendizaje Chamilo.

\section{Tabla 4}

Resultados de la prueba de hipótesis para la diferencia entre los grupos de control y experimental en las capacidades técnicas

\begin{tabular}{|c|c|c|c|c|c|}
\hline \multicolumn{6}{|c|}{ Capacidades técnicas } \\
\hline \multirow[b]{2}{*}{ Fase } & \multirow[b]{2}{*}{ Grupo } & \multirow[b]{2}{*}{ Media } & \multirow{2}{*}{$\begin{array}{l}\text { Desviación } \\
\text { estándar }\end{array}$} & \multicolumn{2}{|c|}{ U de Mann Whitney } \\
\hline & & & & $\mathbf{U}$ & $p$-valor \\
\hline \multirow{2}{*}{ Pretest } & Control & 10.55 & 1.701 & 167.000 & 0.383 \\
\hline & Experimental & 10.00 & 1.076 & & \\
\hline \multirow{2}{*}{ Postest } & Control & 10.75 & 1.372 & 0.000 & 0.000 \\
\hline & Experimental & 34.90 & 3.640 & & \\
\hline
\end{tabular}

Fuente: Prueba de competencias del módulo de Ofimática, postest
Decisión. Como en el pretest de las capacidades técnicas la prueba U de Mann Whitney no indicó diferencias significativas entre los grupos de control y experimental; pero, en el postest, dio ${ }^{* *} p<$ .01 , al .000 de error se concluye que la utilización del entorno virtual Chamilo influye significativamente en el logro de capacidades técnicas del módulo de Ofimática en los estudiantes.

Según la figura 4, en el pretest de las capacidades técnicas, la mediana del grupo de control (11) fue 1 punto más que la mediana del grupo experimental (10), no habiendo diferencias significativas; en cambio, en el postest, la mediana del grupo experimental (36) superó en 25 puntos a la mediana del grupo de control (11).

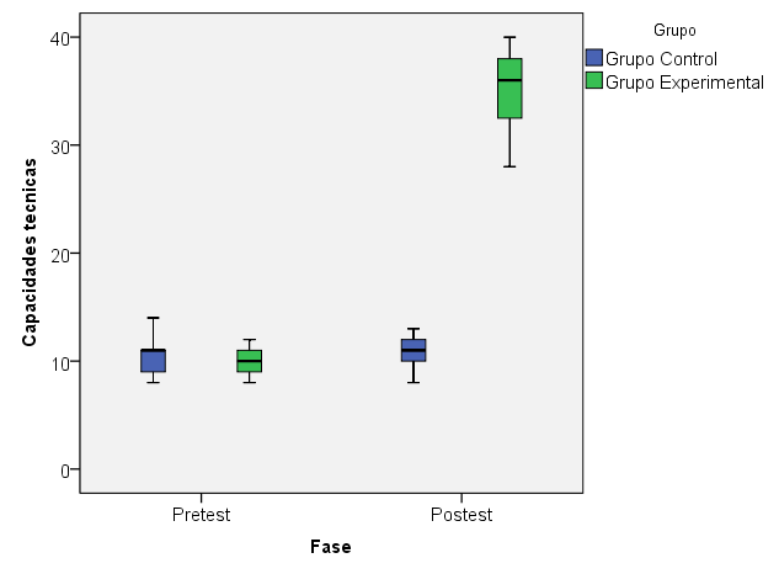

Figura 4. Diagrama de dispersión para la variable logro de capacidades técnicas del módulo de Ofimática en los grupos de control y experimental, pretest y postest.

\section{Hipótesis específicas 2}

$\mathrm{H}_{1}$ : La utilización del entorno virtual Chamilo influye significativamente en el logro de capacidades comunicacionales del módulo de Ofimática en los estudiantes.

$\mathrm{H}_{0}$ : La utilización del entorno virtual Chamilo no influye significativamente en el logro de capacidades comunicacionales del módulo de Ofimática en los estudiantes

Los resultados de la prueba $U$ de Mann Whitney para la diferencia entre los grupos de control y experimental indicaron que, en el 
pretest de las capacidades comunicacionales (tabla 5), no hubo diferencias significativas ( $p>.05$ ) entre los grupos; por el contrario, en el postes, la prueba indicó que hubo una diferencia muy significativa $\left({ }^{* *} p<.01\right)$, como consecuencia de la utilización del entorno virtual Chamilo.

\section{Tabla 5}

Resultados de la prueba de hipótesis para la diferencia entre los grupos de control y experimental en las capacidades comunicacionales

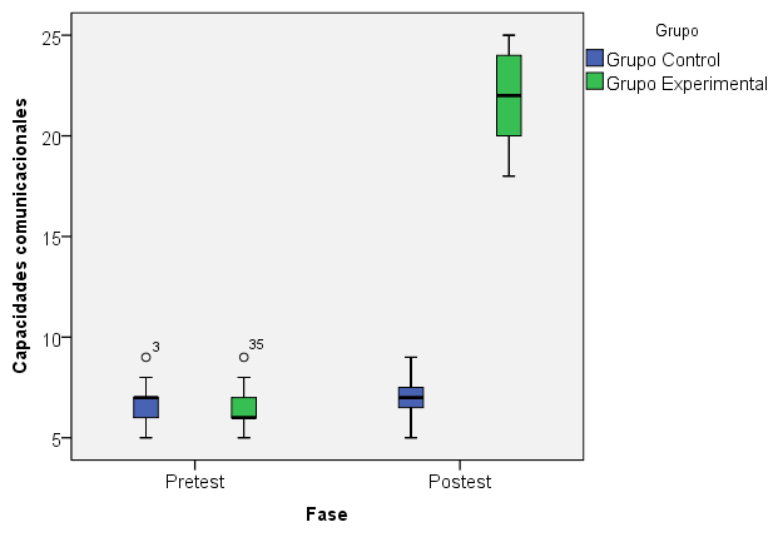

\begin{tabular}{|c|c|c|c|c|c|}
\hline \multicolumn{6}{|c|}{ Capacidades comunicacionales } \\
\hline \multirow[b]{2}{*}{ Fase } & \multirow[b]{2}{*}{ Grupo } & \multirow[b]{2}{*}{ Media } & \multirow{2}{*}{$\begin{array}{l}\text { Desviación } \\
\text { estándar }\end{array}$} & \multicolumn{2}{|c|}{ U de Mann Whitney } \\
\hline & & & & U & $p$-valor \\
\hline \multirow{2}{*}{ Pretest } & Control & 6.60 & 0.995 & 174.000 & 0.495 \\
\hline & Experimental & 10.00 & 6.45 & & \\
\hline \multirow{2}{*}{ Postest } & Control & 7.10 & 1.071 & 0.000 & 0.000 \\
\hline & Experimental & 22.10 & 2.360 & & \\
\hline
\end{tabular}

Figura 5. Diagrama de dispersión para la variable logro de capacidades comunicacionales del módulo de Ofimática en los grupos de control y experimental, pretest y postest.

\section{Hipótesis específica 3}

Fuente: Prueba de competencias del módulo de Ofimática, postest

$\mathrm{H}_{1}$ : La utilización del entorno virtual Chamilo influye significativa-

Decisión. Considerando que en el pretest capacidades comunicacionales la prueba $U$ de Mann Whitney no indicó diferencias significativas entre los grupos de control y experimental y, en el postes, dio ${ }^{* *} p<.01$, al .000 de error se concluye que la utilización del entorno virtual Chamilo influye significativamente en el logro de capacidades comunicacionales del módulo de Ofimática en los estudiantes del instituto.

En la figura 5 se observa que, en el pretest de las capacidades comunicacionales, la mediana del grupo de control (7) fue 1 punto más que la mediana del grupo experimental (6), no habiendo diferencias significativas; en cambio, en el postest, la mediana del grupo experimental (22) superó en 15 puntos a la mediana del grupo de control (7). mente en el logro de capacidades personales y sociales del módulo de Ofimática en los estudiantes. $\mathrm{H}_{0}$ : La utilización del entorno virtual Chamilo no influye significativamente en el logro de capacidades personales y sociales del módulo de Ofimática en los estudiantes.

Los resultados de la prueba U de Mann Whitney para la diferencia entre los grupos de control y experimental indicaron que, en el pretest de las capacidades personales y sociales (tabla 6 ), no hubo diferencias significativas $(p>.05)$ entre ambos grupos; pero, en el postes, la prueba indicó que hubo una diferencia muy significativa $\left({ }^{* *} p<.01\right)$, como consecuencia del uso de Chamilo.

\section{Tabla 6}

Resultados de la prueba de hipótesis para la diferencia entre los grupos de control y experimental en las capacidades personales y sociales

\begin{tabular}{|c|c|c|c|c|c|}
\hline \multicolumn{6}{|c|}{ Capacidades personales } \\
\hline \multirow[b]{2}{*}{ Fase } & \multirow[b]{2}{*}{ Grupo } & \multirow[b]{2}{*}{ Media } & \multirow{2}{*}{$\begin{array}{l}\text { Desviación } \\
\text { estándar }\end{array}$} & \multicolumn{2}{|c|}{ U de Mann Whitney } \\
\hline & & & & $\mathbf{U}$ & $p$-valor \\
\hline \multirow{2}{*}{ Pretest } & Control & 10.55 & 0.999 & 159.000 & 0.277 \\
\hline & Experimental & 10.20 & 1.240 & & \\
\hline \multirow{2}{*}{ Postest } & Control & 11.05 & 1.276 & 0.000 & 0.000 \\
\hline & Experimental & 35.40 & 4.441 & & \\
\hline
\end{tabular}

Fuente: Prueba de competencias del módulo de Ofimática, postest 
El entorno virtual Chamilo en el desarrollo de las competencias del módulo de ofimática en estudiantes del instituto "Norbert Wiener" de Lince

Decisión. Considerando que en el pretest de las capacidades personales y sociales la prueba $U$ de Mann Whitney no indicó diferencias significativas entre los grupos de control y experimental $\mathrm{y}$, en el postest, dio ${ }^{* *} \mathrm{p}<.01$, al .000 de error se concluye que la utilización del entorno virtual Chamilo influye significativamente en el logro de capacidades personales y sociales del módulo de Ofimática en los estudiantes del IES "Norbert Wiener" del distrito de Lince.

La figura 6 permite observar que, en el pretest de las capacidades personales y sociales, la mediana del grupo de control (11) fue 1 punto más que la mediana del grupo experimental (10), no habiendo diferencias significativas; en cambio, en el postest, la mediana del grupo experimental (37) superó en 26 puntos a la mediana del grupo de control (11).

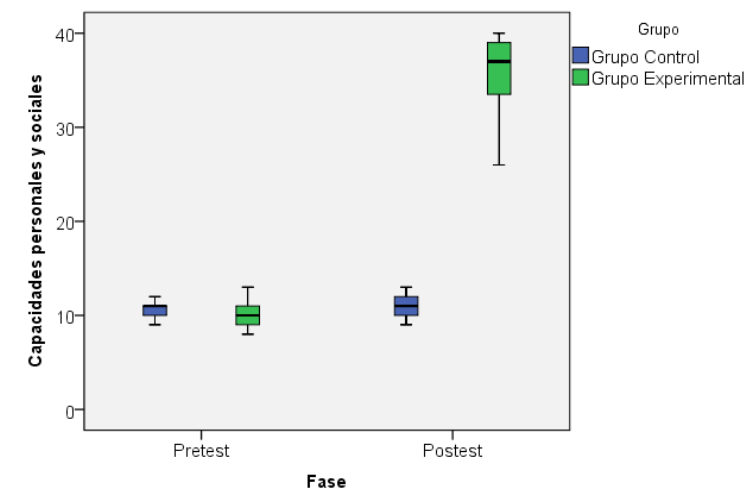

Figura 6. Diagrama de dispersión para la variable logro de capacidades personales y sociales del módulo de Ofimática en los grupos de control y experimental, pretest $y$ postest.

\section{DisCUSión Y CONCLUSIONES}

El uso de las plataformas virtuales o entornos virtuales en la docencia, se ha venido dando en forma paulatina en todos los niveles educativos, las universidades y los institutos superiores no son ajenos a su uso, el cual conlleva a que los docentes realicen cambios en sus procedimientos metodológicos, para incluir estos en su labor pedagógica. Los ordenadores, proyectores, aplicaciones informáticas enfocadas a la docencia, aplicaciones de gestión de estudiantes, pizarras electrónicas, sistemas de comunicación online, entre otros, son solo algunos ejemplos introducidos en la ense- ñanza, que se actualizan cada día y demandan la especialización inmediata, para que puedan utilizarse de forma pertinente en beneficio del aprendizaje de los estudiantes de cualquier nivel, por lo que los entornos virtuales de aprendizaje resultan un escenario óptimo para promover el uso de nuevas herramientas de enseñanza que permitan el conocimiento y uso instrumental de aplicaciones informáticas y, mediante estas, favorecer la adquisición de habilidades cognitivas para el manejo de información hipertextual y multimedia, así como el desarrollo de una actitud crítica y reflexiva para la valoración de la información y las herramientas tecnológicas disponibles, lo cual conlleva además al logro de las competencias en los estudiantes como se ha contrastado en este estudio al haber obtenido como resultado $\mathrm{p}=.000$, aseverando que el entorno virtual Chamilo influye significativamente en el logro de competencias del módulo de Ofimática, resultados similares a Bosco, (2004) al indicar que los entornos virtuales favorecen el aprendizaje autónomo y cooperativo, Ardura y Zamora (2014) coinciden en que al $74 \%$ de los estudiantes les parece adecuado el uso de una plataforma en las clases presenciales $y$ un $34 \%$ manifiesta que su motivación hacia el estudio aumenta.

La actitud que tengan los estudiantes frente a la tecnología, y la aceptación que estos le brinden a la incorporación de estas herramientas tecnológicas en su proceso de aprendizaje y además la motivación y competencia pedagógica del docente y los recursos utilizados por él, son importantes para alcanzar los logros de aprendizaje deseados, hecho que ha sido contrastado en nuestro estudio al evidenciar que el entorno virtual Chamilo influye significativamente en el desarrollo de capacidades técnicas, resultados que se comparte con Maldonado (2012) al comprobar que los estudiantes de la Universidad de Córdova consideran que las plataformas tecnológicas son una alternativa de formación y desarrollo de competencias, y brindan la oportunidad de apertura a la participación en programas de teleformación. Pérez y Saker, (2013) manifiestan que los estudiantes tienen una actitud positiva en el uso de la plataforma virtual de un $74.2 \%$, al contribuir está en el aumento de su rendimiento académico. 
Los resultados de la prueba U de Mann Whitney dieron también $\mathrm{p}=.000$, indicando que el entorno virtual Chamilo influye significativamente en el logro de las capacidades comunicacionales, siendo éstas el conjunto de tareas, conocimientos, habilidades, actitudes y valores que ayudan en la construcción de significados compartidos entre los miembros de una organización (Reyes, 2004 y Trelles, 2009). Las competencias comunicativas no se reducen al manejo instrumental del lenguaje (buena expresión oral y escrita) o manejo ágil de nuevas tecnologías de comunicación; sino que son conceptos integrales cuyo desarrollo es importante para que la persona pueda interactuar y desarrollarse socialmente, lo cual se ha podido visualizar en este estudio sobre el uso del entorno virtual Chamilo, que tiene una incidencia en los estudiantes, siendo estos influenciados positivamente en sus capacidades comunicacionales, pues el docente participa directamente en el proceso de enseñanza, gracias a que la plataforma virtual propicia la relación docente-estudiante; para Pérez y Saker, (2013) indican que el 100\% de los estudiantes refieren que les resulta de utilidad en el desarrollo de los cursos, el uso de la plataforma virtual, ya que facilita la comunicación con sus pares y con el docente.

El uso de las tecnologías no solo puede permitir el desarrollo cognitivo, sino debe buscar integrar competencias personales y sociales, como se ha podido verificar en este estudio en el desarrollo de estas competencias, al haber obtenido $\mathrm{p}=.000$, los estudiantes aprenden a relacionarse con las personas de su entorno, aprenden a valorarse para mejorar su interacción con los demás, coincidiendo con lo comprobado por Fernández (2009) que los entornos virtuales ayudan en la formación colaborativa y cooperativa, es decir, favorecen la adaptación del sujeto a su entorno.

Para estudios posteriores sería relevante realizar una comparación con otros entornos virtuales de uso libre similares a Chamilo, para verificar si estos brindan las mismas bondades o difieren entre ellos o proporcionan un mayor bagaje de herramientas para el docente y estudiantes.

Adicionalmente también sería importante evaluar la usabilidad y accesibilidad del entorno virtual
Chamilo, con aquellos usuarios que tienen más de un año aplicando esta plataforma.

\section{REFERENCIAS BIBIOGRÁFICAS}

Alvites-Huamaní, C. y Bayona, C. (2014) El módulo didáctico de la pizarra digital interactiva mejora el desarrollo de la comprensión tectora de los estudiantes de 5to. grado de primaria de la I.E. 14502, Caserio, Coyona-piura. Recuperado de: http://reposital.cuaed.unam.mx:8080/jspui/ bitstream/123456789/4218/1/VE14.355.pdf

Ardila, J., Ruíz, E. y Castro, I. (2015) Estudio comparativo de sistemas de gestión del aprendizaje: Moodle, Atuto, Claroline, Chamilo y Universidad de Boyacá. Revista Academia y Virtualidad, 8 (1), 54-65.

Ardura, D. y Zamora, A. (2014) ¿Son útiles entornos virtuales de aprendizaje en la enseñanza de las ciencias secundaria? Evaluación de una experiencia en la enseñanza y el aprendizaje de la Relatividad. Revista Eureka sobre Enseñanza y Divulgación de las Ciencias, 11 (1), 83-93.

Arnold, R. y Schüssler, I. (2001). Entwicklung des kompetenzbegriffs und seine bedeutung für die berufsbildung und für die berufsbildungsforschung in: G. Franke (Ed.), Komplexität und kompetenz. Ausgewählte fragen der kompetenzforschung. Bielefeld: Bertelsmann.

Bates, T. (A.W.) (2000). Managing Technological Change. Strategies for College and University Leaders. San Francisco: Jossey-Bass.

Benitez, D. Cruces, E. y Sarrión, M. (2011) El papel de la plataforma virtual de enseńanza en la docencia presencial de asignaturas de estadística. Revista de Formación e Innovación Educativa Universitaria, 4 (1), 1-12.

Bosco, A. (2004). Sobre los nuevos entornos virtuales de enseńanza y aprendizaje Quaderns Digitalis. Recuperado de: http://www.quadernsdigitals.net/datos/hemeroteca/r_1/ nr_558/a_7900/7900.pdf

Chamilo (s.f) Manual del Profesor-Chamilo 1.10. Recuperado de: https:/github.com/chamilo/docs/raw/ master/1.10/es/teacher/chamilo-1.10-guia-profesor.pdf

Dellepiane, P. (2013) Las tutorías en la Universidad: propuesta de formación en entornos virtuales de aprendizaje. Campus Virtuales, 2(2), 66-74.

Escalante, P., Patrón, R. y Argüelles, L. (2011) Mejora de las competencias tecnológicas del docente en la educación media superior. Revista Iberoamericana para la investigación y el desarrollo educativo, 1 (1) 91-109.

Fernández, R. (2009). Factores antecedentes en el uso de Entornos Virtuales de Formación y su efecto sobre el Desempeño docente. Valencia.

Ferreira, A. y Sanz, C. (2012) Musa un modelo de evaluación de entornos virtuales de enseñanza y aprendizaje. Aplicación a un caso de estudio. Revista Iberoamericana

Figueras, S., Capllonch, M., Blázquez, D. y Monzoníz, N. 
El entorno virtual Chamilo en el desarrollo de las competencias del módulo de ofimática en estudiantes del instituto "Norbert Wiener" de Lince

(2016) Competencias básicas y educación física: estudios e investigación. Apunts. Educación Física y Deportes, 123 (1) 34-43.

Forestello, R. y Arónica, S. (2013) Evolución de la plataforma e-ducativa en la Facultad de Ciencias Económicas de la Universidad Nacional de Córdoba, Virtualidad, Educación y Ciencia, 7 (4), 45-59.

Hernández, R., Fernández, C. y Batista, P. (2010). Metodología de la investigación.(6a ed.). México: McGraw Hill.

Ibáñez, J., Delgado, C. \& Aylett, R. (2006) Virtual Enviroments: A Multiy-disciplinary Field, 7 (2),

MAD. (2005). Ayudante técnico de informática de la Junta de andalucía: Temario. Volumen II (2a ed.). Sevilla: MAD.

Maldonado, G. (2012). Actitudes con respecto al uso de la plataforma tecnológica de Tele formación Moodle: El caso de los estudiantes de la Facultad de Ciencias de la Educación. Universidad de Córdoba, España.

Marqués, P. (2000) Los docentes: funciones, roles, competencias necesarias, formación. Recuperado de: http://www. uaa. $\mathrm{mx} /$ direcciones/dgdp/defaa/descargas/docentes_funciones.pdf

Mora-Valentín y Ortiz-De-Urbina-Criado,M. (2015) ¿Cómo fomentar el desarrollo de competencias en la formación on-line? Una experiencia en la asignatura de dirección estratégica. Recuperado de: http://dx.doi.org/10.14201/ eks201516290108

Ospina, D. (2009). ¿Qué es un ambiente virtual de aprendizaje? Recuperado el 12 de diciembre de 2012 de: http:// aprendeenlinea.udea.edu.co/banco/html/ambiente_virtual_ de_aprendizaj e/,

Pazo, C. y Tejada, J. (2012) Las competencias profesionales en educación física. Retos. Nuevas tendencias en educación física, deporte y recreación, 22 (2), 5-8.

Peña, M. y Avendaño. B. (2006). Evaluación de la implementación del aula virtual en una institución de Educación Superior. Suma Psicológica, 13(2),173-192.

Pèrez, M. y Saker, A. (2013) Importancia del uso de las plataformas virtuale en formación superior para favorecer el cambio de actitud hacia las TIC; estudio de caso: Universidad del Magdalena, Colombia. Revista Iberoamericana de Evaluaciòn Educativa, 6 (1), 153-166.

Reyes, M. (2004). El Desarrollo de Competencias Comunicativas: uno de los Principales Retos en la Educación Superior a Distancia. Latin Educa 2004, Primer Congreso Latino Americano de Educacion a distancia (pp. 42-62). Mexico: Facultad de Estudios Superiores Acatlán, UNAM.

Rodrigues, M., Simóes, E. y Ferreira, M. (2013) Competencias sociales en la estrategia de desarrollo de carrera. Apuntes de Psicología, 31 (1), 93-99.

Salinas, M. (2011). Entornos virtuales de aprendizaje, tipos, modelos didácticos y el rol del docente. Buenos Aires, Argentina.

Trelles, I. (2009). Introducción. En: Trelles, I., Comunicación Organizacional (p. vi). La Habana: Félix Varela.
Universidad Politécnica de Valencia-UPV (2006) Plan de acciones para la convergencia Europea, Guía docente de la UPV, criterios para su elaboración. Recuperado de: http:// www.aqu.cat/doc/doc_52850666_1.pdf

Van Arcken, C. (2010). Definición de estándares de requisitos tecnológicos y científicos de los prestadores de servicios de salud en Colombia para ofertar un servicio de osteopatía. Bogotá: Universidad Nacional de Colombia.

Valenzuela-Zambrano, B. y Pérez-Villalobos, M. (2013) Aprendizaje autorregulado a través de la plataforma virtual Moodle. Educ. 16 (1), 66-79. Recuperado de: http://www. redalyc.org/pdf/834/83428614009.pdf 